\title{
Regulation of the Endoplasmic Reticulum Stress by BIP/GRP78 is involved in Meiotic Maturation of Porcine Oocytes In Vitro
}

\author{
Hyo-Jin Park ${ }^{1, *}$, Jae-Young Park ${ }^{1,2, *}$, Jin-Woo Kim¹, Seul-Gi Yang1, \\ Jae-Min Jung ${ }^{1}$, Min-Ji Kim¹, Joung Jun Park ${ }^{3}$ and 'Deog-Bon Koo ${ }^{1}$ \\ ${ }^{1}$ Dept. of Biotechnology, College of Engineering, Daegu University, Gyeongsan 38453, Republic of Korea \\ ${ }^{2}$ Saewha Hospital, Dongnae, Busan 47822, Republic of Korea \\ ${ }^{3}$ Animal Reproduction \& Biotechnology Center, Myung-Poom Hanwoo Consulting, Gangwon 25232, Republic of Korea
}

\begin{abstract}
In the present study, we investigated the role of binding immunoglobulin protein/glucose-regulated protein, 78-kDa (BIP/GRP78)-regulated endoplasmic reticulum (ER)-stress on meiotic maturation and cumulus cells expansion in porcine cumulus-oocyte complexes (COCs). Previously, it has been demonstrated that unfolded protein response (UPR)related genes, such as molecules involved in ER-stress defense mechanisms, were expressed in matured oocytes and cumulus cells during in vitro maturation (IVM) of porcine oocytes. However, BIP/GRP78-mediated regulation of ER stress in porcine oocytes has not been reported. Firstly, we observed the effects of knockdown of BIP/GRP78 (an UPR initiation marker) using porcine-specific siRNAs (\#909, \#693, and \#1570) on oocyte maturation. Among all siRNAs, siRNA \#693 significantly reduced the protein levels of UPR marker proteins (BIP/GRP78, ATF4, and P90ATF6) in porcine COCs observed by Western blotting and immunofluorescence analysis. We also observed that the reduction of BIP/GRP78 levels by siRNA\#693 significantly inhibited the meiotic maturation of oocytes (siRNA \#693: $32.5 \pm 10.1 \%$ vs control: $77.8 \pm 5.3 \%$ ). In addition, we also checked the effect of ER-stress inhibitors, tauroursodeoxycholic acid (TUDCA, $200 \mu \mathrm{M})$ and melatonin $(0.1 \mu \mathrm{M})$, in BIP/ GRP78-knockdown oocytes. TUDCA and melatonin treatment could restore the expression levels of ER-stress marker proteins (BIP/GRP78, p-eIF2 $\alpha$, eIF2 $\alpha$, ATF4, and P90ATF6) in siRNA \#693-transfected matured COCs. In conclusion, these results demonstrated that BIP/GRP78-mediated regulation of UPR signaling and ER stress plays an important role in in vitro maturation of porcine oocytes.
\end{abstract}

Key words : ER stress, BIP/GRP78, UPR, In vitro maturation, Porcine oocyte

\section{INTRODUCTION}

The endoplasmic reticulum (ER), a cellular organelle, is well known as a modulator of protein folding and calcium homeostasis. As a result of various ER functions, ER stress is induced by the accumulation of unfolded proteins in the ER lumen (Raab et al., 2009). To inhibit the ER stress,
UPR (Unfolded protein response) signaling is activated for cell survival. Under ER stress, three UPR transducers, protein kinase-like ER kinase (PERK), activating transcription factor 6 (ATF6), and inositol-requiring enzyme 1 (IRE1), dissociate from their binding partner, Glucose-Regulated Protein, 78-kDa (BIP/GRP78), which then binds to the unfolded proteins (Wu \& Kaufman, 2006). First, to prevent

\footnotetext{
Manuscript received October 15, 2017, Received in revised form November 17, 2017, Accepted December 4, 2017

${ }^{\dagger}$ Corresponding Author : Deog-Bon Koo, Department of Biotechnology, College of Engineering, Daegu University, Gyeongsan 38453, Republic of Korea. Tel: +82-53-850-6557, Fax: +82-53-850-6559, E-mail: dbkoo@daegu.ac.kr

* The first two authors contributed equally to this work.

This is an Open Access article distributed under the terms of the Creative Commons Attribution Non-Commercial License (http:// creativecommons.org/licenses/by-nc/3.0) which permits unrestricted non-commercial use, distribution, and reproduction in any medium, provided the original work is properly cited.
} 
new protein synthesis in the stressed ER, the activated PERK phosphorylates eukaryotic initiation factor $2 \alpha$ (eIF2 $\alpha)$ to inhibit translation initiation. However, phosphorylated eIF $2 \alpha$ subsequently induces the expression of activating transcription factor 4 (ATF4). Second, P90ATF6 is cleaved by site-1 protease (S1P) and site-2 protease (S2P) in the Golgi compartment and converted into activated P50ATF6. Third, phosphorylation of IRE1 induces the splicing of $\mathrm{x}$ box binding protein 1 (XBP1), which converts it into a more potent transcription factor that regulates the transcription of ER chaperones. IRE1 signaling inhibits protein synthesis and induces ER-associated degradation (ERAD) by the ubiquitin-dependent proteasomal pathway (Teske et al., 2011a). Finally, repetitive and persistent ER stress induces the activation of CCAAT-enhancer-binding protein homologous protein (CHOP), which regulates the transcription of apoptotic factors during ER stress (Namba \& Kodama, 2015).

$\mathrm{BIP/GRP78}$ is a major chaperone protein for protein quality control in the ER (Wang et al., 2009). In particular, $\mathrm{BIP} / \mathrm{GRP} 78$ is a critical regulator that controls the activation of ER-transmembrane signaling mechanisms, such as ER-stress-induced activation of three pathways of UPR. Further, it plays an important role in cell proliferation, differentiation, and protection of the inner cell mass of blastocysts from apoptosis during embryonic development (Luo et al., 2006). BIP/GRP78-mediated activation of three UPR signal pathways is also involved in the reproduction system, such as apoptosis regulation in granulosa cells (Farinola et al., 2007), and cell cycle arrest in lung cancer cells (Zhu et al., 2015). In addition, many studies demonstrated that the inhibition of ER stress is involved in meiotic maturation, parthenogenetic embryonic development in vitro (Zhang et al., 2012b), ovarian granulosa cell apoptosis (Yang et al., 2013), embryonic development of mice (Zhang et al., 2012a), and granulosa cell apoptosis during follicular atresia in goat (Lin et al., 2012). However, the role of BIP/GRP78-related ER stress or activation of UPR signaling pathways in in vitro maturation (IVM) of porcine oocytes has not been reported.

Therefore, we investigated the effect of BIP/GRP78regulated ER stress on porcine oocyte maturation. The objective of the current study was to determine whether BIP/GRP78-regulated ER stress affects meiotic maturation and UPR signaling gene expression in the porcine oocytes.

\section{MATERIALS AND METHODS}

\section{Chemicals}

Unless indicated otherwise, all chemicals in this study were purchased from Sigma Aldrich Korea (St. Louis, MO, USA).

\section{In vitro maturation (IVM) of oocyte}

We performed IVM as described by Kim et al. (2016), but with slight modification. Pig ovaries were collected from a local slaughterhouse and transported to our laboratory at $35^{\circ} \mathrm{C}$ in $0.9 \%$ saline supplement with $75 \mu \mathrm{g} / \mathrm{mL}$ potassium penicillin G. Immature cumulus-oocyte-complexes (COCs) between 3 and $6 \mathrm{~mm}$ in diameter were aspirated into a $10 \mathrm{~mL}$ syringe from ovary follicles by using an 18-gauge needle. Subsequently, undamaged aspirated COCs with similar quality cytoplasm and with oocytes surrounded by cumulus cells were selected by using mouth pipettes. The selected COCs were washed three times in TL-HEPES medium, and approximately $50 \mathrm{COCs}$ were matured in 500 $\mu \mathrm{L}$ of IVM medium at $38.5^{\circ} \mathrm{C}$ and under $5 \% \mathrm{CO}_{2}$ in air. The IVM medium was NCSU-23 medium with $0.57 \mathrm{mM}$ cysteine, $10 \%$ porcine follicular fluid, $10 \mathrm{ng} / \mathrm{mL}$ epidermal growth factor (EGF), $10 \mathrm{ng} / \mathrm{mL} \beta$-mercaptoethanol, $10 \mathrm{IU} /$ $\mathrm{mL}$ pregnant mare serum gonadotropin (PMSG), and 10 $\mathrm{IU} / \mathrm{mL}$ human chorionic gonadotropin (hCG). After culturing for $22 \mathrm{~h}$, COCs were washed three times and then cultured further in IVM medium without PMSG and hCG for $22 \mathrm{~h}$. And then, we collected the matured porcine COCs at the $44 \mathrm{~h}$ after IVM. 


\section{Assessment of meiotic maturation}

After $44 \mathrm{~h}$ of IVM, meiotic maturation was distinguished by nuclear stages. Oocytes were denuded by pipetting in TL-HEPES medium containing $0.1 \%$ hyaluronidase, washed in PVA-PBS and mounted on microscope slides. The samples were fixed for 3 days in acetic acid/ethanol (1: $3, \mathrm{v} / \mathrm{v})$, stained with $1 \%$ acetic orcein (v/v) for $5 \mathrm{~min}$. Meiotic stage of the samples was evaluated under a microscope (Leica, Solms, Germany).

\section{RNA extraction and reverse transcription (RT) -} PCR

Total RNA were extracted from porcine COCs using TRIzol reagent (Invitrogen, CA) according to the manufacturer's instructions. Extracted RNA was quantified by a NanoDrop spectrophotometer (ACTgene, Piscataway, NJ). Each cDNA was synthesized from the aliquots $(1 \mu \mathrm{g} / \mu \mathrm{L})$ of total RNA with AccuPower ${ }^{\circ}$ RT-PCR Premix (Bioneer, Korea). PCR was carried out using AccuPower ${ }^{\circledR}$ PCR Premix (Bioneer) containing specific primers: $B I P / G R P 78$; GGTGGGCAAACAAAGACATT (sense) and CGCTGGT CAAAGTCTTCTCC (antisense). Specific primer sequences were designed using the NCBI database.

\section{Protein extraction and western blotting}

Following the $44 \mathrm{~h}$ IVM period, lysates of $30 \mathrm{COCs}$ were prepared in protein lysis buffer PRO-PREP (iNtRON Biotechnology, Seoul, Korea) with centrifugation at 13,000 rpm for $10 \mathrm{~min}$ at $4{ }^{\circ} \mathrm{C}$. Sample lysates were separated on $12 \%$ polyacrylamide gel by using sodium dodecyl sulfatepolyacrylamide gel electrophoresis (SDS-PAGE) and then transferred onto nitrocellulose membranes (Pall Corporation, NY, USA). The membranes were blocked by incubation with 5\% skim milk in Tris-buffered saline (TBS) containing $0.1 \%$ Tween 20 overnight at $4{ }^{\circ} \mathrm{C}$. Membranes were then incubated with the appropriate primary antibody: antibody anti-GRP78 (1 : 2,000; Santa Cruz Biotechnology, CA, USA), anti-ATF4 (1 : 5,000; Santa Cruz), anti-P90
ATF6 (1 : 4,000; Novus Biologicals, USA), anti- $\beta$-ACTIN (1 : 3,000; Santa Cruz), anti-p-eIF2 $\alpha$ (1:2,000; cell signaling) and anti-eIF2 $\alpha$ (1: 2,000; cell signaling) antibodies. Following incubation, membranes were washed and incubated with secondary antibody HRE-conjugated anti-mouse/ rabbit IgG (Thermo Fisher Scientific, Rockford, IL, USA) for $3 \mathrm{~h}$ at room temperature. Antibody binding was detected by using a chemiluminescent system (Bight ECL Kit, Advansta, Menlo Park, CA, USA) according to the manufacturer's instructions. Band intensities were quantified by using Image J software (National Institutes of Health, MD).

\section{Transfection of siRNA for porcine BIP/GRP78}

Three predesigned potential BIP/GRP78 siRNAs were chemically synthesized by GenePharma (Shanghai, China). Maturated COCs were transfected with BIP/GRP78 siRNA (100 nM) and negative control siRNA (25 nM) using lipofectamine 3000 according to the manufacturer's instructions. Sequences of porcine BIP/GRP78 and negative control siRNAs were as-following : \#909: CCU UCU CAC CAU UGA UAA UTT (sense), AUU AUC AAU GGU GAG AAG GTT (antisense); \#693: GGG AAA GAA GGU UAC UCA UTT (sense), AUG AGU AAC CUU CUU UCC CTT (antisense); \#1570: GCC UCU GAU AAU CAG CCA ATT (sense), UUG GCU GAU UAU CAG AGG CTT (antisense); negative control siRNA: UUC UCC GAA CGU GUC ACG UTT (sense), ACG UGA CAC GUU CG AGA ATT (antisense).

\section{Immunofluorescence (IF) staining}

After IVM $44 \mathrm{~h}$ after siRNA treatment, COCs in M II (44 h) were washed with $0.3 \%$ PVA-PBS and fixed in $4 \%$ (v/v) paraformaldehyde and $2.5 \%(\mathrm{v} / \mathrm{v})$ glutaraldehyde solution for $1 \mathrm{~h}$ at room temperature. Next, COCs were transferred to a permeabilization solution $(0.2 \%$ Triton X-100) at room temperature for $1 \mathrm{~h}$ and then incubated in $0.3 \%$ PVA-PBS overnight at $4{ }^{\circ} \mathrm{C}$. After blocking overnight at $4{ }^{\circ} \mathrm{C}$ in $0.1 \%$ PVA-PBS containing $1 \% \mathrm{BSA}$, the COCs were 
incubated with anti-BIP/GRP78 (sc-1050; Santa cruz) diluted $1: 500$ at $4{ }^{\circ} \mathrm{C}$ overnight. After incubation, the COCs reacted with the secondary antibody, FITC-conjugated goat anti-rabbit IgG (Santa Cruz Biotechnology), diluted 1 : 1000 in $0.3 \%$ PVA-PBS for $2 \mathrm{~h}$ at room temperature. DAPI reagent $(2 \mathrm{mg} / \mathrm{mL})$ was used to stain the nuclei. Immunoreactivity was observed under a Zeiss LSM 700 confocal microscope (Carl Zeiss, Oberkochen, Germany).

\section{Statistical analysis}

All percentage data and data sets were subjected to arsine transformation and expressed as the mean \pm standard deviation (SD). And all values of Western blot experiments were presented as the mean \pm standard error of the mean (SEM). The results were analyzed using a one-way ANOVA followed by Bonferroni's Multiple Comparison Test and using $t$-tests. All data were performed using the GraphPad Prism 5.0 software package (San Diego, CA, USA). Differences were considered significant at ${ }^{*} p<0.05$, ${ }^{* *} p<0.01$, and ${ }^{* * *} p<0.001$.

\section{RESULTS}

\section{Changes of UPR marker proteins expressions in} COCs after siRNA BIP/GRP78 treatment

To observe the effects of BIP/GRP78 on oocyte maturation, we used specific siRNA (\#693, \#1570 and \#909) of BIP/GRP78 for pig (data not shown). After siRNA \#693, \#1570 and \#909 treatment, we chose the most appropriate depending on transfection efficiency. Over all, we determined the siRNA \#693 of BIP/GRP78 for use in subsequent experiments.

Changes in BIP/GRP78 fluorescence expression in negative control of siRNA and siRNA \#693 transfected COCs were investigated by immunofluorescence staining. As shown in Fig. 1B BIP/GRP78 fluorescence intensity was lower $(p<0.001)$ in siRNA \#693 of BIP/GRP78 transfected COCs than other treated groups (Fig. 1B). In addition, to investigate the mRNA and protein levels of BIP/GRP78, we performed RT-PCR analysis and Western blotting, respectively. As a result, mRNA and protein levels of BIP/ GRP78 significantly decreased $(p<0.001)$ in the BIP/GRP78 siRNA \#693 treated COCs compared with control and negative siRNA treated groups (Fig. 1C and 1D). Moreover, ATF4 and P90ATF6 protein levels were reduced the in siRNA \#693 transfected COCs. These results indicated that the reduction of BIP/GRP78 expression by siRNA \#693 reduced the UPR marker proteins expression of COCs at $44 \mathrm{~h}$ of IVM in porcine oocytes.

\section{Verification of ER-stress by decreasing of BIP/} GRP78 expression on porcine oocyte maturation

To confirm the induced ER-stress by reduction of BIP/ GRP78 gene expression on porcine oocyte maturation, we used the IVM medium supplemented with ER-stress inhibitor, TUDCA $(200 \mu \mathrm{M})$ and melatonin $(0.1 \mu \mathrm{M})($ Fig. 2A: mimetic diagram of experiment methods). First, we investigated the meiotic maturation by acetic-orcein staining (Table 1). The meiotic maturation was reduced $(p<0.05)$ in COCs of siRNA \#693 treated groups compared with control and other treated groups (Table 1). As expected, meiotic maturation significantly recovered $(p<0.001$; siRNA \#693: $32.5 \pm 10.1 \%$ vs siRNA \#693+TUDCA: $73.9 \pm 3.1 \%$ and siRNA \#693+Mela: $77.2 \pm 2.9 \%$ ) in the COCs of TUDCA or melatonin treatment after pre-transfected siRNA \#693. Additionally, we investigated the UPR marker proteins (BIP/GRP78, p-eIF2 $\alpha$, eIF2 $\alpha$, ATF4 and P90ATF6) in COCs of TUDCA or melatonin treatment after pre-transfected siRNA \#693, respectively. As shown in the Fig. 2B and $2 \mathrm{C}$, expression levels of most UPR marker proteins were significantly recovered in TUDCA or melatonin treated COCs after siRNA \#693 transfection $(p<0.001)$. These results suggested that induction of ER-stress by siRNA \#693 for inhibition of BIP/GRP78 gene expression was affected the meiotic maturation in porcine COCs during IVM. 
A)

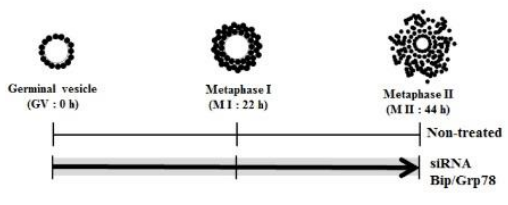

B)

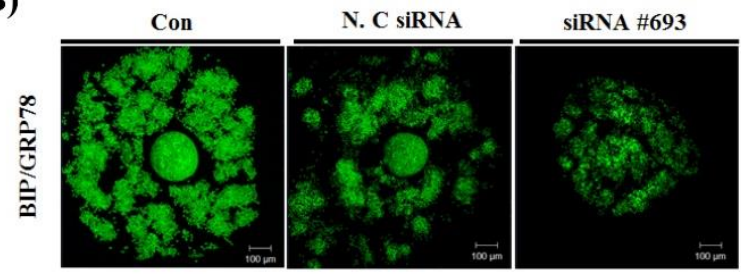

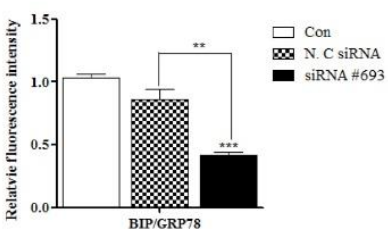

C)
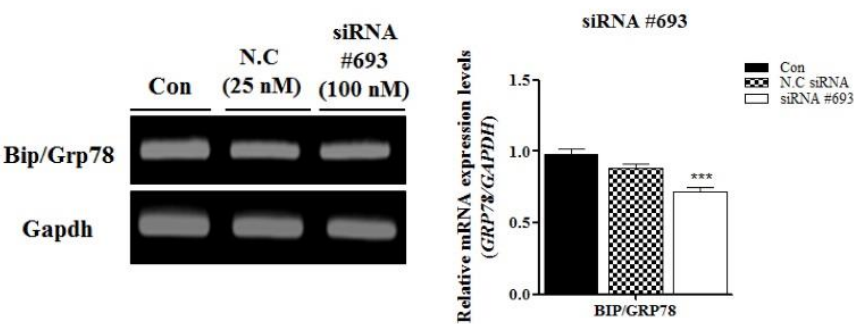

D)

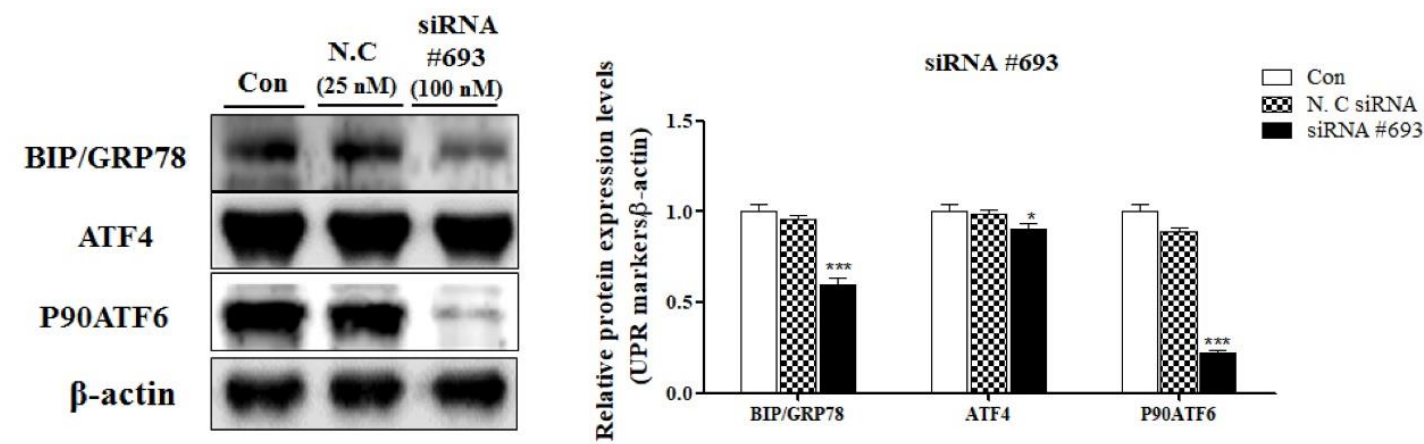

Fig. 1. Effect of siRNA \#693-mediated knockdown of BIP/GRP78 on in vitro maturation (IVM, 44 h) of porcine oocytes. (A) Schematic representation of the experimental method involved in siRNA \#693-mediated knockdown of BIP/GRP78 in porcine oocytes. (B) Expression levels of BIP/GRP78 in control and siRNA \#693-transfected COCs were observed by immunofluorescence staining and analyzed by a confocal microscope. Scale bars $=100$ $\mu \mathrm{m}$. (control; untreated and N.C.; negative control siRNA). (C) mRNA levels of BIP/GRP78 (UPR initiation marker) in in vitro maturing control and siRNA \#693-transfected COCs were measured by RT-PCR analysis. Relative change in the levels of BIP/GRP78 was calculated by normalizing the signals of BIP/GRP78 with respective GAPDH signals. (D) Expression levels of BIP/GRP78, ATF4, and P90ATF6 were compared in siRNA \#693transfected COCs at the M II (44 h) stage of pig oocyte maturation by Western blot analysis. Relative change in the levels of UPR marker proteins was obtained by normalizing the signals with respective $\beta$-actin signals. Histograms represent values of densitometry analysis calculated by using Image $\mathbf{J}$ software. Data are represented as mean \pm SEM or SD of three independent experiments (30 COCs). Differences were considered significant at ${ }^{*} p<0.05,{ }^{* *}$ $p<0.01$, and ${ }^{* * *} p<0.001$.

\section{DISCUSSION}

The purpose of the present study is to examine the effect of BIP/GRP78-knockdown (using siRNA \#693) on ER stress and meiotic maturation in porcine oocytes during
IVM. Previously, ER-molecular chaperones, GRP78 and GRP94, have been identified in the germinal vesicle $(\mathrm{GV})$, germinal vesicle breakdown (GVBD), metaphase I (M I), and metaphase II (M II) stages of porcine oocytes, by 2dimensional gel electrophoresis (2-DE) (Ellederova et al., 
A)

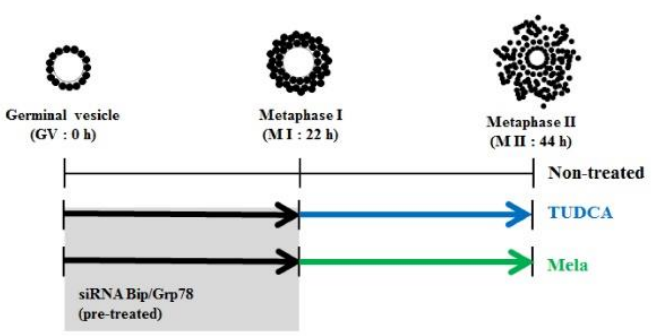

B)

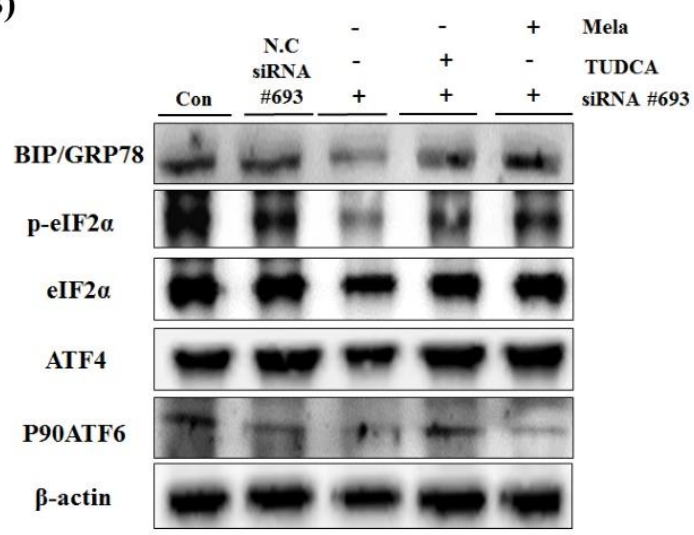

C)
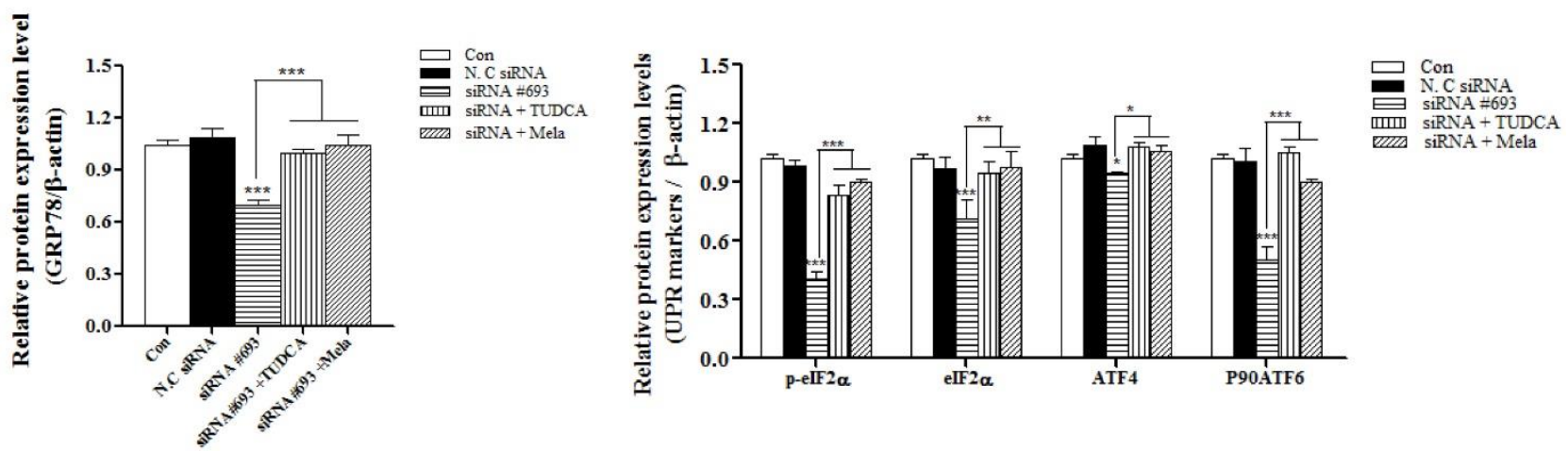

Fig. 2. Effects of siRNA \#693-mediated knockdown of BIP/GRP78 on activation of UPR signaling during pig oocyte maturation. (A) Schematic diagram of the experimental method showing the timeline of TUDCA or melatonin treatment of COCs, which were pre-transfected with siRNA \#693. (B-C) Western blot analysis of BIP/GRP78, peIF2 $\alpha$, eIF2 $\alpha$, ATF4, and P90ATF6 in TUDCA or melatonin-treated COCs (Metaphase I; M I), which were pretransfected with siRNA \#693 for $22 \mathrm{~h}$. Relative change in the levels of UPR marker proteins was obtained by normalizing the band intensities to $\beta$-actin. Histograms represent values of densitometry analysis calculated by Image $\mathrm{J}$ software. Data are represented as mean $\pm \mathrm{SEM}$ of three independent experiments $(30 \mathrm{COCs})$. Differences were considered significant at ${ }^{*} p<0.05,{ }^{* *} p<0.01$, and ${ }^{* * *} p<0.001$.

2004). The roles of ER stress or the regulation of UPR signaling mechanisms has been reported in mouse embryos. BIP/GRP78 gene is key regulator for ER stress response. In addition, ER stress regulation is involved in proliferation, differentiation and cellular apoptosis through the enhancement of UPR marker protein expression. However, there is no report on the directly role and expression of BIP/GRP78 in the in vitro maturated oocyte and cumulus cells oocyte complex (COCs) of porcine. As shown in Fig. $1 \mathrm{~B}$ and $1 \mathrm{C}$, protein levels of BIP/GRP78 were decreased in
siRNA\#693 treated COCs compared with negative siRNA and/or control group. These results suggest that BIP/ GRP 78 expression is correlated with the regulation ER-stress and UPR signaling pathways in COCs during IVM progression. Because of the importance of BIP/GRP78 in ER stress, we investigated the BIP/GRP78-mediated regulation of UPR signaling in porcine oocytes and its role in oocyte maturation.

BIP/GRP78 plays an important role in signal transduction via formation of different complexes with other pro- 
Table 1. Effects of siRNA \#693-mediated knockdown of BiP/GRP78 on induction of ER stress and meiotic maturation of pig oocytes during IVM

\begin{tabular}{cccccc}
\hline \hline & $\begin{array}{c}\text { No. of oocytes } \\
\text { examined }\end{array}$ & GV & GVBD & M I & \% of oocytes (n) \\
\cline { 3 - 6 } & 145 & $3.9 \pm 4.5(3)$ & $2.3 \pm 1.8(4)$ & $16.1 \pm 5.4(26)^{\mathrm{a}}$ & $77.8 \pm 5.3(112)^{\mathrm{a}}$ \\
Con & 143 & $1.5 \pm 2.0(2)$ & $4.3 \pm 5.5(6)$ & $28.1 \pm 7.5(41)^{\mathrm{b}}$ & $66.1 \pm 2.8(94)^{\mathrm{b}}$ \\
N.C siRNA & 162 & $3.9 \pm 2.6(5)$ & $9.6 \pm 9.2(11)$ & $54.0 \pm 11.7(90)^{\mathrm{d}}$ & $32.5 \pm 10.1(56)^{\mathrm{d}}$ \\
siRNA \#693 & 126 & $3.8 \pm 3.1(6)$ & $5.1 \pm 6.3(9)$ & $17.2 \pm 6.9(27)^{\mathrm{a}}$ & $73.9 \pm 3.1(84)^{\mathrm{a}}$ \\
siRNA \#693+TUDCA & 155 & $1.4 \pm 2.9(2)$ & $2.6 \pm 3.5(5)$ & $18.8 \pm 5.4(29)^{\mathrm{a}}$ & $77.2 \pm 2.9(119)^{\mathrm{a}}$ \\
\hline
\end{tabular}

The table represents the $\%$ of oocytes in different stages of oocyte maturation in control and siRNA \#693-transfected COCs with or without TUDCA $(200 \mu \mathrm{M})$ and melatonin $(0.1 \mu \mathrm{M})$ treatment. Data are represented as mean \pm SD of three independent experiments. ${ }^{\mathrm{a} \sim \mathrm{d}}$ superscripts denote a significant difference among groups $(p<0.05)$.

teins on the cell surface and in ER lumen (Mukherjee \& Soto, 2011). BIP/GRP78 (binding immunoglobulin protein: BiP, also known as glucose-regulated protein: GRP78) is an endoplasmic reticulum (ER) chaperone protein, which is encoded by HSPA5 and contributes to protein folding in the ER compartment. Recently, many reports have shown that the BIP/GRP78 is involved in female reproduction system, oviduct, and early embryonic development. Previous report has shown that the maternal contribution of HSP90b1 (encodes GRP94) is critical for the development of murine zygotes and is involved in specific aspects of the first mitosis of murine zygotes (Audouard et al., 2011).

Various studies have shown that regulation of ER stress and UPR signaling play critical roles in maturation of oocytes and cumulus cells, and early embryonic development. The BIP/GRP78 (HSPA5) regulates the ER-stress-induced UPR signaling, which is essential for protecting the inner cell mass from apoptosis in early embryonic development of mouse (Luo \& Mao, 2006). Chemical chaperones reduced ER stress in a mouse model of type- 2 diabetes $(\mathrm{Oz}-$ can et al., 2006). In addition, ER stress in porcine COCs impaired pentraxin3 (PTX3) secretion from cumulus cells and delayed embryonic development during the blastocyst stage (Wu et al., 2012). Our result demonstrated that siRNA \#693 against BIP/GRP78 inhibited the expression of UPR signal activators, ATF4 and P90ATF6, in porcine COCs during IVM (Fig. 1D). Further, PERK-mediated phosphorylation of eIF2 $\alpha$ during ER stress represses new protein synthesis by blocking the initiation codon. Phosphorylated eIF $2 \alpha$ (p-eIF2 $\alpha$ ) also induces expression of a transcription activator, ATF4, which regulates integrated stress response (Teske et al., 2011b). In the present study, we found that the PERK/p-eIF2 $\alpha /$ ATF4 signaling pathway is downregulated in siRNA \#693-transfected COCs (Fig. $2 \mathrm{~B}$ and $2 \mathrm{C})$.

Next, we hypothesized that if BIP/GRP78-regulated ERstress delayed maturation of porcine oocytes, inhibition of ER stress should restore the maturation process. Melatonin is a well known oxidative stress scavenger (Agarwal et al., 2005) and has antioxidant properties (Poston et al., 2011). We used melatonin as an inhibitor of ER stress in porcine oocytes during maturation as described previously (Carloni et al., 2014). We also used TUDCA as another ER-stress inhibitor. As shown in the Table 1 and Fig. 2, TUDCA and melatonin treatment could restore the ER-stress-induced inhibition of meiotic maturation of porcine COCs. Concur- 
rently, expression of UPR markers, including BIP/GRP78, was significantly increased in TUDCA and melatonintreated COCs.

In conclusion, our results emphasized that BIP/GRP78 regulates ER stress during oocyte maturation, as well as plays key roles in in vitro maturation of porcine oocytes.

\section{ACKNOWLEDGEMENTS}

This work was supported by grants from the Next-Generation BioGreen 21 Program (PJ01117604), Cooperative Research Program for Agriculture Science and Technology Development (PJ01269503) and the Bio-industry Technology Development Program (316037-4) through the Rural Development Administration and the Ministry of Agriculture, Food and Rural Affairs, Republic of Korea.

\section{REFERENCES}

Agarwal A, Gupta S, Sharma RK (2005) Role of oxidative stress in female reproduction. Reprod Biol Endocrinol : RB\&E 3:28.

Audouard C, Le Masson F, Charry C, Li Z, Christians ES (2011) Oocyte-targeted deletion reveals that hsp90b1 is needed for the completion of first mitosis in mouse zygotes. PloS one 6:e17109.

Carloni S, Albertini MC, Galluzzi L, Buonocore G, Proietti F, Balduini W (2014) Melatonin reduces endoplasmic reticulum stress and preserves sirtuin 1 expression in neuronal cells of newborn rats after hypoxia-ischemia. J Pineal Res 57:192-199.

Ellederova Z, Halada P, Man P, Kubelka M, Motlik J, Kovarova $H$ (2004) Protein patterns of pig oocytes during in vitro maturation. Biol Reprod 71:1533-1539.

Farinola MA, Gown AM, Judson K, Ronnett BM, Barry TS, Movahedi-Lankarani S, Vang R (2007) Estrogen receptor alpha and progesterone receptor expression in ovarian adult granulosa cell tumors and Sertoli-Leydig cell tumors. Int J Gynecol Pathol 26:375-382.

Kim JW, Park HJ, Chae SK, Ahn JH, DO GY, Choo YK, Park JJ, Jung BD, Kim SU, Chang KT, Koo DB (2016) Ganglioside GD1a promotes oocyte maturation, furthers preimplantation development, and increases blastocyst quality in pigs. J Reprod Dev 62:249-255.

Lin P, Yang Y, Li X, Chen F, Cui C, Hu L, Li Q, Liu W, Jin $Y$ (2012) Endoplasmic reticulum stress is involved in granulosa cell apoptosis during follicular atresia in goat ovaries. Mol Reprod Dev 79:423-432.

Luo S, Mao C, Lee B, Lee AS (2006) GRP78/BiP is required for cell proliferation and protecting the inner cell mass from apoptosis during early mouse embryonic development. Mol Cell Biol 26:5688-5697.

Mukherjee A, Soto C (2011) Role of calcineurin in neurodegeneration produced by misfolded proteins and endoplasmic reticulum stress. Curr Opin Cell Biol 23: 223-230.

Namba T, Kodama R (2015) Avarol induces apoptosis in pancreatic ductal adenocarcinoma cells by activating PERK-eIF2alpha-CHOP signaling. Mar Drugs 13:23762389.

Ozcan U, Yilmaz E, Ozcan L, Furuhashi M, Vaillancourt E, Smith RO, Gorgun CZ, Hotamisligil GS (2006) Chemical chaperones reduce ER stress and restore glucose homeostasis in a mouse model of type 2 diabetes. Science 313:1137-1140.

Poston L, Igosheva N, Mistry HD, Seed PT, Shennan AH, Rana S, Karumanchi SA, Chappell LC (2011) Role of oxidative stress and antioxidant supplementation in pregnancy disorders. Am J Clin Nutr 94:1980S-1985S.

Raab MS, Breitkreutz I, Tonon G, Zhang J, Hayden PJ, Nguyen T, Fruehauf JH, Lin BK, Chauhan D, Hideshima T, Munshi NC, Anderson KC, Podar K (2009) Targeting PKC: a novel role for beta-catenin in ER stress and apoptotic signaling. Blood 113:1513-1521.

Teske BF, Baird TD, Wek RC (2011a) Methods for analyzing eIF2 kinases and translational control in the un- 
folded protein response. Methods in Enzymology 490: 333-356.

Teske BF, Wek SA, Bunpo P, Cundiff JK, McClintick JN, Anthony TG, Wek RC (2011b) The eIF2 kinase PERK and the integrated stress response facilitate activation of ATF6 during endoplasmic reticulum stress. Mol Biol Cell 22:4390-4405.

Wang M, Wey S, Zhang Y, Ye R, Lee AS (2009) Role of the unfolded protein response regulator GRP78/BiP in development, cancer, and neurological disorders. Antioxid Redox Signal 11:2307-2316.

Wu J, Kaufman RJ (2006) From acute ER stress to physiological roles of the unfolded protein response. Cell Death Differ 13:374-384.

Wu LL, Russell DL, Norman RJ, Robker RL (2012) Endoplasmic reticulum (ER) stress in cumulus-oocyte complexes impairs pentraxin-3 secretion, mitochondrial membrane potential (DeltaPsi m), and embryo develop- ment. Mol Endocrinol 26:562-573.

Yang Y, Lin P, Chen F, Wang A, Lan X, Song Y, Jin Y (2013) Luman recruiting factor regulates endoplasmic reticulum stress in mouse ovarian granulosa cell apoptosis. Theriogenology 79:633-639 e631-633.

Zhang JY, Diao YF, Kim HR, Jin DI (2012b) Inhibition of endoplasmic reticulum stress improves mouse embryo development. PloS one 7:e40433.

Zhang JY, Diao YF, Oqani RK, Han RX, Jin DI (2012a) Effect of endoplasmic reticulum stress on porcine oocyte maturation and parthenogenetic embryonic development in vitro. Biol Reprod 86:128.

Zhu J, Chen M, Chen N, Ma A, Zhu C, Zhao R, Jiang M, Zhou J, Ye L, Fu H, Zhang X (2015) Glycyrrhetinic acid induces G1 phase cell cycle arrest in human nonsmall cell lung cancer cells through endoplasmic reticulum stress pathway. Int J Oncol 46:981-988. 